\title{
EVOLUÇÃO MOTORA INCOMUM NA DOENÇA DO REFLUXO GASTROESOFÁGICO
}

\author{
Unusual motor disorder on GERD
}

\author{
Antônio Moreira MENDES-FILHO, Andréa R.G.V. MEDEIROS, Rayanne Maria B. da SILVEIRA, \\ Vanessa O. M. GUEDES, Ilanna N. L. RODRIGUES, Simone Leal BARBOSA
}

Trabalho realizado na Clínica Digest e Brasil.

DESCRITORES - Acalásia esofágica. Refluxo gastroesofágico. Espasmo esofagiano difuso. Universidade Estadual do Piauí, Teresina, PI,

RESUMO - Introdução - Demonstra-se que os distúrbios motores esofágicos como o espasmo esofageano difuso podem progredir para acalásia. Também é conhecida a associação entre a doença do refluxo gastroesofágico e alguns destes distúrbios. Relato do caso - Paciente com queixa de dor torácica, disfagia e perda de peso há dois meses. A primeira endoscopia digestiva alta sugeriu apenas dismotilidade esofágica. Posteriormente, realizou manometria esofágica que evidenciou alterações em corpo compatíveis com espasmo esofageano difuso, sem alterações no esfíncter esofageano inferior. $\mathrm{Na}$ oportunidade iniciou o tratamento com pantoprazol e nifedipina que se estendeu por oito meses; no entanto manteve-se sintomática. Após suspender medicação por 10 dias, realizou pH-metria esofageana prolongada constatando-se refluxo gastroesofágico patológico combinado e refluxo proximal, sendo submetida ao tratamento com esomeprazol e mantida a nifedipina. Após dois meses, com a persistência dos sintomas, realizou-se nova endoscopia que evidenciou estase alimentar e manometria esofágica com aperistalse completa de corpo. Neste momento, optou-se por cardiomiotomia a Heller com fundoplicatura laparoscópica. A endoscopia do terceiro mês do pós-operatório evidenciou fundoplicatura bem ajustada ao aparelho. No seguimento a médio prazo ela permanece assintomática. Conclusão - Existe correlação entre os distúrbios motores esofágicos e a doença do refluxo gastroesofágico, e o tratamento cirúrgico para corrigir a acalásia e o refluxo gastroesofágico ao mesmo tempo, pode ser indicado em casos selecionados.

\section{Correspondência:}

Antonio Moreira Mendes Filho, e-mail: moreira-filho@uol.com.br

Fonte de financiamento: não há Conflito de interesses: não há

Recebido para publicação: 22/09/2009 Aceito para publicação: 14/06/2010

HEADINGS - Esophageal achalasia. Gastroesophageal reflux. Diffuse esophageal spasm.
ABSTRACT - Background - It is shown that esophageal motor disorders, such as diffuse esophageal spasm, may progress to achalasia. It is also known a link between gastroesophageal reflux disease and some of these motor disorders. Case report - Patient complaining of chest pain, dysphagia and weight loss for two months. The first endoscopy suggested only motility disorders. Esophageal manometry was done and showed changes in body compatible with diffuse esophageal spasm, without changes in lower esophageal sphincter. Treatment with pantoprazole and nifedipine for eight months was done, but the patient remained symptomatic. After stopping medication for 10 days, an esophageal $\mathrm{pH}$-metry was realized and showed gastro-esophageal reflux. She was submitted to treatment with esomeprazole and nifedipine. After two months, with persistence of the symptoms, a new endoscopy showed food stasis, and esophageal manometry body esophageal aperistalsis. At this point, surgical treatment (Heller cardiomyotomy with fundoplication) was done. Endoscopy of the third postoperative month showed fundoplication well adjusted to the endoscope. In follow-up, patient remained asymptomatic. Conclusion There is a correlation between esophageal motor disorders and gastroesophageal reflux disease, and surgical treatment to correct achalasia and gastroesophageal reflux at the same time, may be indicated in selected cases.

\section{INTRODUÇÃO}

calásia é um distúrbio motor primário do esôfago que acomete
a musculatura lisa, caracterizado por relaxamento incompleto
do esfíncter inferior do esôfago e perda da peristalse no 
e também com a doença do refluxo gastroesofágico. Esse espasmo é caracterizado manometricamente por proporção maior de $30 \%$ de complexos de deglutição conduzidos de forma aperistáltica ${ }^{8}$. Além disso, pode ocorrer também a presença de ondas terciárias ou retrógradas levando à incoordenação da contratilidade do esôfago ${ }^{10}$, e determinando sintomas como dor retroesternal e/ou disfagia.

A doença do refluxo gastroesofágico é afecção crônica de prevalência elevada em nosso meio? ${ }^{9}$ A presença constante do conteúdo gastroduodenal (refluxato) em contato com a mucosa esofágica determina presença de ondas de condução de baixa amplitude e falhas de condução ${ }^{7}$. Apesar do distúrbio motor mais frequentemente associado ser o esôfago em quebra-nozes ${ }^{13}$, essa afecção é dita também (em menor proporção) como possível causa do espasmo esofageano difuso, podendo ser primário ou associado à doença do refluxo gastroesofágico ${ }^{3}$.

\section{RELATO DO CASO}

Mulher de 42 anos procurou atendimento médico com queixas de dor torácica, disfagia e perda de peso, há dois meses. Tinha realizado endoscopia digestiva alta que demonstrou dismotilidade e manometria evidenciou alterações motoras compatíveis com espasmo esofageano difuso, com esfíncter inferior do esôfago preservado quanto à tonicidade e capacidade de relaxamento. $\mathrm{Na}$ oportunidade, a paciente foi medicada com pantoprazol $40 \mathrm{mg} / \mathrm{dia}$ e nifedipina $20 \mathrm{mg} / \mathrm{dia}$, mas não referiu melhora clínica. Após suspensão da medicação por 10 dias, foi indicada $\mathrm{pH}$-metria esofageana prolongada que mostrou refluxo gastroesofágico patológico combinado e refluxo proximal (Figura 1 ).

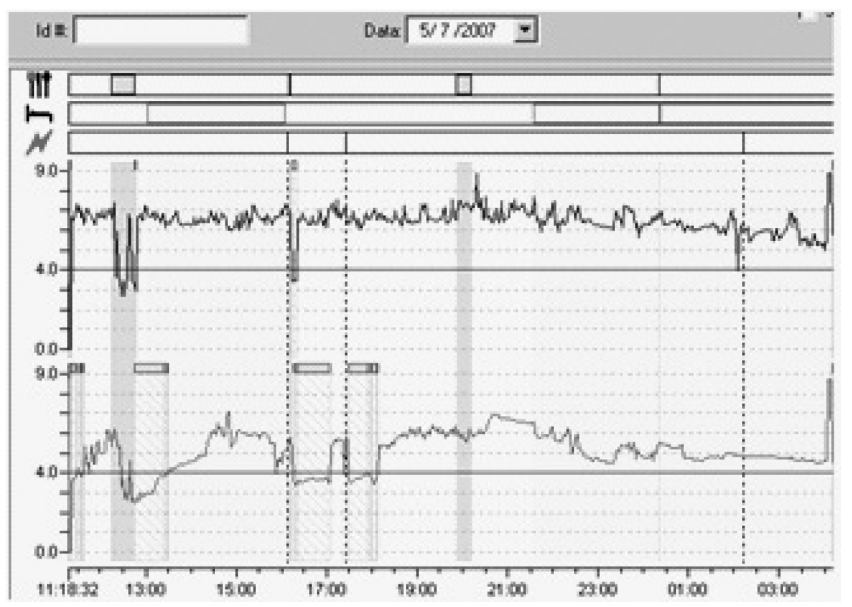

FIGURE 1 - pH-metria esofágica constatando a presença de refluxo gastroesofágico patológico combinado e refluxo proximal

O tratamento clínico foi mantido com esomeprazol em dose dobrada mantendo-se a nifedipina. Em nova reavaliação (dois meses após este período), referia sintomas persistentes, queixando-se principalmente da disfagia e emagrecimento. Foi realizada nova endoscopia digestiva alta (Figura 2) que demonstrou discreta dilatação do diâmetro esofágico e resíduos alimentares em seu interior.

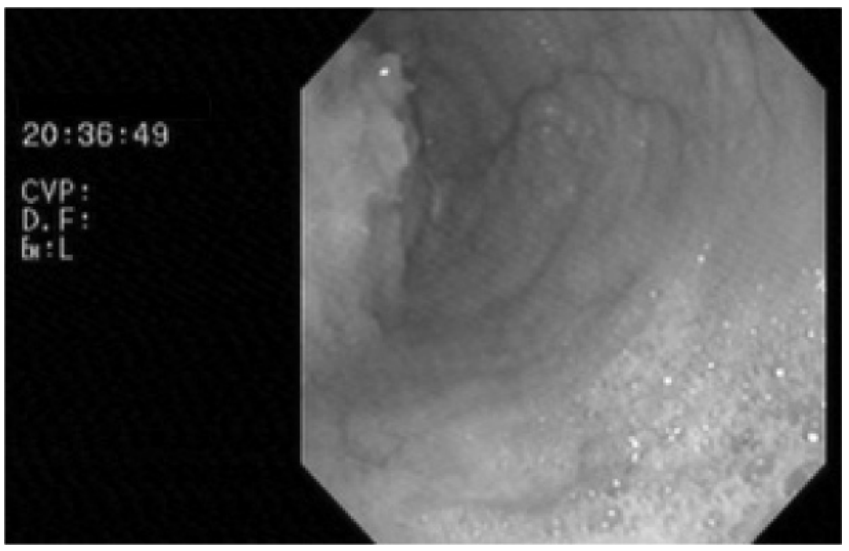

FIGURE 2 - Endoscopia digestiva alta demonstrando discreta dilatação do corpo esofágico e resíduos alimentares em seu interior

Nova manometria foi realizada evidenciando aperistalse no corpo esofágico (Figura 3), não tendo a sonda ultrapassado o esfíncter inferior do esôfago.

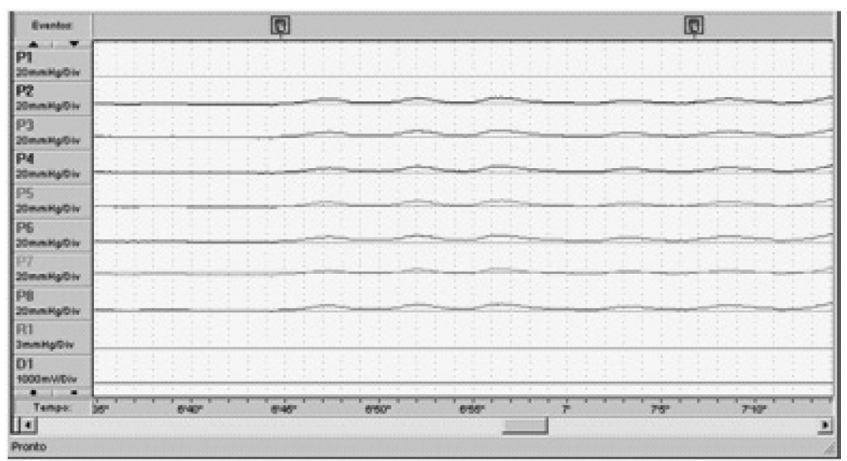

FIGURE 3 - Manometria evidenciando completa aperistalse no corpo esofágico

Baseado nesses dados clínicos, tratamento cirúrgico com cardiomiotomia a Heller laparoscópica com fundoplicatura foi realizado. A paciente teve boa evolução pós-operatória e após 18 meses apresentavase somente com raros episódios de disfagia, tendo recuperado o seu peso inicial.

\section{DISCUSSÃO}

O termo acalásia (do grego; a = ausência de, calasia = abertura) foi empregado pela primeira vez em 1914 por Hurst ${ }^{5}$, que, levando em consideração a facilidade com que se introduziam bugias no esôfago 
destes pacientes, sugeriu ser distúrbio primário não decorrente de espasmos, mas, sim, de falta de relaxamento da cárdia ${ }^{7}$. Embora não reflita com precisão a totalidade das alterações motoras presentes, esse vocábulo vem se mantendo na literatura como descritor desta afecção.

O substrato fisiopatológico consiste em alterações da inervação intrínseca esofágica, com diminuição dos neurônios do plexo mioentérico ${ }^{2}$. A inervação inibitória, mediada via óxido nítrico é a mais comprometida; como a via excitatória colinérgica está preservada, ocorre geralmente aumento da pressão basal do esfíncter inferior do esôfago ${ }^{4}$.

Nos últimos anos, a acalásia tida como idiopática tem sido associada como possível complicação da doença do refluxo gastroesofágico de longa evolução ${ }^{14}$ e também de distúrbios motores esofágicos primários . Anggiaansah et al. ${ }^{1}$ relataram um caso que evoluiu de esôfago em quebra-nozes para acalásia. Vantrappen et al. ${ }^{15}$, por sua vez, relataram seis pacientes com distúrbio motor inespecífico com esta progressão. Foi encontrado nessa revisão, um relato de caso muito semelhante ao aqui descrito ${ }^{12}$ de um paciente portador de doença do refluxo gastroesofágico que teve como complicação, a longo prazo, o desenvolvimento de espasmo esofageano difuso e acalásia; em comparação ao caso que aqui foi relatado, este paciente era mais jovem (24 anos) e do sexo masculino.

Especificamente em relação ao espasmo esofageano difuso, alguns autores ${ }^{3}$ propuseram classificação como idiopático e associado à doença do refluxo gastroesofágico. O mecanismo envolvido seria o já mencionado anteriormente de exposição crônica da mucosa esofágica ao refluxato, com o desenvolvimento de hipocontratilidade e distúrbios de condução ${ }^{7}$, que terminariam, ao longo do tempo, por determinar esta condição.

Hayashi et al. ${ }^{3}$, em um ensaio clínico controlado, compararam 11 pacientes com espasmo esofageano difuso associado à doença do refluxo gastroesofágico e 30 de causa idiopática; eles encontraram que na primeira condição a pirose seria o sintoma mais prevalente (com diferença estatística significante), enquanto que disfagia e dor torácica seriam os sintomas mais prevalentes no segundo grupo (sem diferença estatística); ainda segundo estes autores o tratamento com inibidores de bomba de prótons poderia ser eficaz no espasmo esofageano difuso secundário. O estudo de caso exposto aqui não confirma estes achados, pois as queixas mais relevantes eram dor torácica e disfagia (posteriormente a perda de peso), e o tratamento com inibidores da bomba de prótons, mesmo em dose dobrada, não teve resultado significativo.
O tratamento cirúrgico para distúrbios primários de motilidade, como acalásia, espasmo esofageano difuso e esfíncter esofageano hipertensivo é indicado em casos selecionados ${ }^{6}$. Consiste em realizar cardiomiotomia e fundoplicatura parcial. Esta foi a opção escolhida neste caso, até mesmo pelo desenvolvimento da acalásia, e o resultado em 18 meses apresentou-se satisfatório.

\section{CONCLUSÃO}

Existe correlação entre os distúrbios motores esofágicos e a doença do refluxo gastroesofágico e o tratamento cirúrgico, para corrigir a acalásia e o refluxo gastroesofágico ao mesmo tempo, pode ser indicado em casos selecionados.

\section{REFERÊNCIAS}

1. Anggiansah A, Bright NF, McCullagh, Owen WJ. Transition from nutcracker esophagus to achalasia. Dig Dis Sci 1990; 35(9):1162-1166.

2. Goldblum JR, Whyte RI, Orringer MB, Applema HD. Achalasia: a morphologic study of 42 ressected specimens. Am J Surg Pathol. 1994; 18: 327-337.

3. Hayashi H, Mine K, Hosoi M, Tsuchida O, Handa M, Kinukawa N, Andou K, Kubo C. Comparison of the Esophageal Manometric Characteristics of Idiopathic and Reflux-Associated Esophageal Spasm: Evaluation by 24-Hour Ambulatory Esophageal Motility and pH Monitoring. Dig Dis Sci. 2003 Nov; 48 (11): 2124-2131.

4. Holloway RH, Dodds WJ, Helms JF. Integrity of cholinergic innervation to the lower esophageal sphincter in achalasia. Gastroenterology. 1986; 90: 924-929.

5. Hurst AF. Achalasia of the cardia. Quart J Med. 1914; 8: 300-308.

6. Lecont M, Doward R, Gautric M, Dousset B. Surgical management of primary esophageal Motility Disorders. J Chir (Paris). 2008;145 (5): 428-436.

7. Lemme EMO. Distúrbios motores do esôfago. In: Moraes-Filho JPP. Tratado das enfermidades gastrintestinais e pancreáticas. $1^{\text {a }}$ ed. São Paulo: Editora Rocca; 2008. p. 481-498.

8. Machado WM. Espasmo difuso do esôfago. In: Nasi A, Michelsohn $\mathrm{NH}$. Avaliação funcional do esôfago - manometrias e $\mathrm{pH}$-metrias esofágicas. $1^{a}$ ed. São Paulo: Editora Rocca; 2001. p. 112-122.

9. Moraes-Filho JPP, Chinzon D, Eisig J, et al. Prevalence of hertburn and gastroesophageal reflux disease in th urban Brazilian population. Arq Gastroenterol 2005; 42: 444-454.

10. Moretzsohn LD. Distúrbios motores esofágicos não associados a acalasia. In: Domingues G. Esôfago. $1^{\mathrm{a}}$ ed. Rio de Janeiro: Livraria e Editora Rubio; 2005. p. 219-227.

11. Narducci F, Bassotti G, Gaburri M, Morelli A. Transition from nutcracker esophagus to diffuse esophageal spasm. Am J Gastroenterol. 1985; 80(4): 242-244.

12. Robson K, Rosemberg S, Lembo T. Gerd progressing to diffuse esophageal spasm and then to achalasia. 2000;45 (1) : 110-113

13. Silva LFD, Lemme EMO. Esôfago em quebra-nozes - avaliação clínica de 97 pacientes. Arq Gastroenterol. 2000 Out./ Dez.; 37 (4): 217-222.

14. Smart HL, Mayberry JF, Arkinson M. Achalasia following gastroesophageal reflux. J R Soc Med. 1986; 79: 71-73.

15. Vantrappen G, Janssens HOJ, Hellemans HO, Coremans G. Achalasia, diffuse esophageal spasm, and related motility disorders. Gastroenterology 1979; 76: 450-457. 